\title{
Wired-Wireless Converged Passive Optical Network with 4-PAM and Multi-sub-bands FBMC
}

\author{
Hum Nath Parajuli and Eszter Udvary, Member, IEEE
}

\begin{abstract}
Future 5G based passive optical networks (PON) are expected as capable of the simultaneous provision of wired and wireless services for multi-users. In this paper, for the first time, we propose and demonstrate the simultaneous delivery of wired 4-pulse amplitude modulation (4-PAM) and wireless multi-sub-bands filter bank multicarrier (FBMC) signals in one wavelength using one laser source for the future 5G PON. The 4-PAM can be used in cost-efficient intensity modulation direct detection (IM/DD) systems and it provides the double bandwidth efficiency compared to conventional on-off keying (OOK). FBMC is considered as a potential candidate for future wireless 5G due to its high suppression for out of band emissions, which allows combining multiple sub-bands with very narrow band-gaps. Using multi-sub-bands with a narrow band gap, the overall transmission capacity can be increased. In the designed system, the composite wired 4-PAM and wireless multi-sub-bands FBMC signal is generated and transmitted with intensity modulation in optical line terminal (OLT). In the optical network unit $(\mathrm{ONU})$ the wired and wireless signals from the received composite signal are extracted using an electrical square band-pass filter and separately demodulated using digital signal processing techniques. The designed 4-PAM has baseband bandwidth of $4.8 \mathrm{GHz}$ and multi-subbands FBMC consists of 4 sub-bands of $500 \mathrm{MHz}$ each, having very narrow inter-sub-bands gap of $488.28 \mathrm{kHz}$ and the aggregate bandwidth of 2.0015 GHz. The bit error rate (BER) has been evaluated for the performance analysis of the 4-PAM and multi-sub-bands FBMC for two cases (a) separate transmission and (b) composite transmission.
\end{abstract}

Index Terms- passive optical network, filter bank multicarrier, wired-wireless convergence, fifth generation

\section{INTRODUCTION}

The passive optical network (PON) provides the high capacity and flexibility in signal delivery through the fixed access network. PON is considered as an effective solution for $5 \mathrm{G}$ based wireless signals backhauling and fronthauling [1-3]. The future $5 \mathrm{G}$ systems should be capable of supporting multi-services/signals to keep the compatibility with the current legacy wired/wireless services. In this regard, it is important to study and analyze the convergence and delivery of potential $5 \mathrm{G}$ wireless signals with wired signals in the future PON systems.

This work has been carried out within the project FiWiN5G, supported from European Union's Horizon 2020 research and innovation program.

Hum Nath Parajuli is a Marie Curie early stage researcher in Budapest University of Technology and Economics, Budapest, Hungary (e-mail: hum.nath.parajuli@hvt.bme.hu).

Eszter Udvary is an Associate Professor in Budapest University of Technology and Economics, Budapest, Hungary (e-mail: udvary@hvt.bme.hu).
Future 5G networks are expected to provide 1-10 Gbps wireless access to the end users [4-6]. The multicarrier modulation formats are potential solutions to increase the spectral efficiency in future $5 \mathrm{G}$ based wireless communication system. One of the widely studied modulation format in a multicarrier system is orthogonal frequency division multiplexing (OFDM) because of its advantages such as better spectral efficiency and robustness to the fiber optic impairments such as chromatic dispersion (CD) [7, 8]. However, OFDM requires a cyclic prefix $(\mathrm{CP})$ in the overhead to reduce the inter symbol interference (ISI) and inter carrier interference (ICI), which reduces the spectral efficiency. Moreover, large out of band emission of the OFDM subcarriers require large guard bands in multi-sub-bands systems. These problems can be overcome through filter bank multicarrier (FBMC) system [9, 10]. The side lobe suppression of FBMC is about $40 \mathrm{~dB}$ in comparison with OFDM which is about $13 \mathrm{~dB}$ [10]. Sufficient reduction of out of band emission and the combination of the filter banks and offset-QAM (OQAM) leads to no need of the CP overhead. The feature of suppression of side lobes in large extent in FBMC enables asynchronous carrier aggregation very efficiently with a very low effect of interference in comparison with other multi-carrier systems [9-13].

4-pulse amplitude modulation (4-PAM) supports the current intensity modulation and direct detection (IM/DD) system and provides the double bandwidth efficiency compared to the on-off keying (OOK). Due to these benefits, recently huge research interests are shown on this modulation format for cost-effective optical access network design [14-16].

OFDM and FBMC based passive optical network was experimentally demonstrated in [17]. The performance comparison of OFDM and FBMC carrier aggregated signals at mm-wave frequencies was studied with the aggregated bandwidth of less than $1.5 \mathrm{GHz}[10,12]$. These demonstrations show that the FBMC outperforms the OFDM for equivalent design parameters. Adaptively modulated FBMC was also demonstrated in the wired-wireless converged network with the aggregated bandwidth of $1.507 \mathrm{GHz}$ [18]. This demonstration deals with the OFDM and FBMC both modulation formats as a wired/wireless converged system. The convergence of potential $5 \mathrm{G}$ modulation formats such as universal filter multi-carrier (UFDM) and generalized filter multi-carrier (GFDM) as wireless signals and 4-PAM signal as a wired signal in a PON has been demonstrated [19]. This demonstration deals with the single sub-band UFDM and GFDM modulation formats with a very low bandwidth of $1.95 \mathrm{MHz}$ for each modulation format.

All of the above mentioned recent demonstrations of wired/wireless convergence in PON have not been dealt with the convergence of multi-sub-bands FBMC as a wireless and 4-PAM as a 
wired signal. In this paper, we demonstrate the convergence of 4 subbands FBMC as a wireless signal and 4-PAM as a wired signal in a PON. The aggregate bandwidth of the designed 4 sub-bands FBMC signal is $2.0015 \mathrm{GHz}$ with inter-sub-band gap frequency of 488.28 $\mathrm{kHz}$. The bandwidth of the designed 4-PAM baseband signal is 4.8 GHz. The 4-PAM and FBMC sub-bands are extracted and demodulated in the receiver by using digital signal processing (DSP) techniques. The aggregate data rate with 16QAM modulation order for 4 -sub-bands FBMC is $4 \mathrm{Gbps}$ and 4-PAM is $8 \mathrm{Gbps}$. We evaluate the performance of the converged signals by simulating various design parameters using bit error rate (BER) calculations.

The organization of this paper is as follows. In section II, 4-PAM and FBMC multi-sub-bands signal generation method is given. In section III, the description of the implemented system model of optical transmission setup is given. Section IV presents the signal processing methods for received converged signal extraction and demodulation. Section V illustrates the simulation results and discussions. Finally, section VI concludes the paper.

\section{Converged 4-PAM and Multi-Sub-BAnds FBMC Signal GENERATION}

The MATLAB routines are developed to generate offline code for 4-PAM and multi-sub-bands FBMC signals. The baseband 4-PAM signal is generated with $4 \mathrm{GHz}$ bandwidth. The sampling frequency is $32 \mathrm{GS} / \mathrm{s}$ and each 4-PAM symbol is upsampled with 4 samples for each 4-PAM symbol. After this, the root raised cosine (RRC) filter with a roll-off factor of 0.2 is used for pulse shaping.

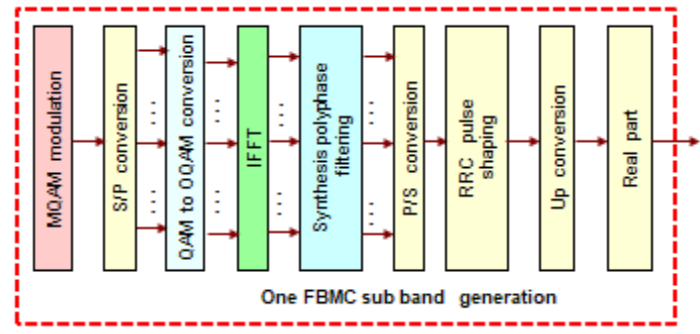

(a)

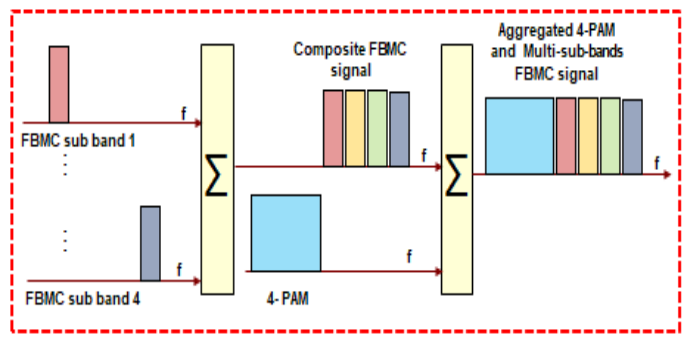

(b)

Fig. 1. Functional block diagram of (a) FBMC signal generation for single sub-band (b) 4 sub-band FBMC signals and 4-PAM signal aggregation.

Fig.1 (a) shows the simplified DSP block diagram for generating single sub-band FBMC signal. First, the input data stream is mapped into $\mathrm{M}$ quadrature amplitude modulation (M-QAM) format, and then it is converted from serial to parallel $(\mathrm{S} / \mathrm{P})$ streams. Then, the $\mathrm{QAM}$ to offset QAM (OQAM) conversion is achieved by making the adjacent symbols with half symbol period offset to each other. After this, inverse fast Fourier transform (IFFT) is applied. Each subcarrier is filtered with well-designed prototype filter (in our case we use prototype filter proposed in [13]), this process is called synthesis polyphase filtering (SPF). After the parallel to serial $(\mathrm{P} / \mathrm{S})$ conversion process, root raised cosine (RRC) filter is applied to optimize the signal to noise ratio (SNR). Then, the baseband FBMC signal is upconverted to the desired carrier frequency. After this, the real part of the signal is taken. In this way, 4 sub-bands are generated and added up to generate 4 sub-bands' composite FBMC signal. The 4-PAM signal is added up with composite FBMC signal to generate multiplexed 4-PAM and 4 sub-bands FBMC signal as shown in Fig. 1 (b).

Table 1. Design parameters of 4 sub-bands FBMC and 4-PAM signals generation

\begin{tabular}{|c|c|c|}
\hline Parameters & \multicolumn{2}{|c|}{ Values } \\
\hline $\begin{array}{c}\text { Modulation } \\
\text { format }\end{array}$ & FBMC & 4 -PAM \\
\hline No. of bits & 32768 & 131072 \\
\hline Bit rate & $4 \mathrm{Gbps}$ & $8 \mathrm{Gbps}$ \\
\hline $\begin{array}{c}\text { No of sub- } \\
\text { bands }\end{array}$ & 4 & 1 \\
\hline $\begin{array}{c}\text { Sub-bands } \\
\text { spacing }\end{array}$ & $\begin{array}{c}488.28 \\
\mathrm{kHz}\end{array}$ & - \\
\hline BW & $\begin{array}{c}2.0015 \\
\mathrm{GHz}\end{array}$ & $\begin{array}{c}4.8 \\
\mathrm{GHz}\end{array}$ \\
\hline $\begin{array}{c}\text { Sampling } \\
\text { frequency }\end{array}$ & \multicolumn{2}{|c|}{$32 \mathrm{GS} / \mathrm{s}$} \\
\hline Time window & \multicolumn{2}{|c|}{$8.192 \mu \mathrm{s}$} \\
\hline
\end{tabular}

The multi-sub-bands FBMC and 4-PAM signals design parameters are given in Table 1. The sampling frequency is $32 \mathrm{GS} / \mathrm{s}$ and each OQAM symbol is upsampled with 64 samples for each OQAM symbol. IFFT/FFT size of 1024 is used. 4 FBMC symbols are created which form one FBMC sub-band. Each sub-band has a bandwidth of $500 \mathrm{MHz} .4$ sub-bands are added up to constitute the composite multi-sub-bands FBMC signal of bandwidth $2.0015 \mathrm{GHz}$ with gap frequency of $488.28 \mathrm{kHz}$ between each sub-band. The slight broadening of bandwidth in the composite FBMC signal is due to the pulse shaping roll-off factor of 0.2. The central frequency of first subband is chosen to be $5.1 \mathrm{GHz}$. With the equal gap frequency of $488.28 \mathrm{kHz}$ between each sub-band, the central frequencies of the second and subsequent sub-bands are $5.6005 \mathrm{GHz}, 6.1010 \mathrm{GHz}$, and $6.6015 \mathrm{GHz}$. Because of the multiplexing in frequency domain the time window of 4-PAM, single-sub-band FBMC and multi-sub-bands signals are identical and equal to $8.192 \mu \mathrm{s}$.

The 4-PAM signal has been broadened up to $4.8 \mathrm{GHz}$ due to the pulse shaping roll-off factor of 0.2. Also, the multi-sub-bands FBMC signal has been broadened and started from $4.8 \mathrm{GHz}$. There is no gap frequency between the 4-PAM and FBMC signal. The total bandwidth of aggregated 4-PAM and multi-sub-bands FBMC from $\mathrm{dc}$ is $6.9015 \mathrm{GHz}$. The total number of bits used for the case of FBMC is 32768 and for the case of 4-PAM is 131072. For 4-PAM and for each sub-bands generation in FBMC, uncorrelated bits sequences are used. Fig. 2 (a) shows the offline generated spectra of 4 sub-bands FBMC signal and (b) shows the aggregated 4-PAM and FBMC signal. As shown in Fig. 2 (a) the sidelobes suppression of FBMC sub-band is about $40 \mathrm{~dB}$ which allows tight packing of subbands without significant interference. 


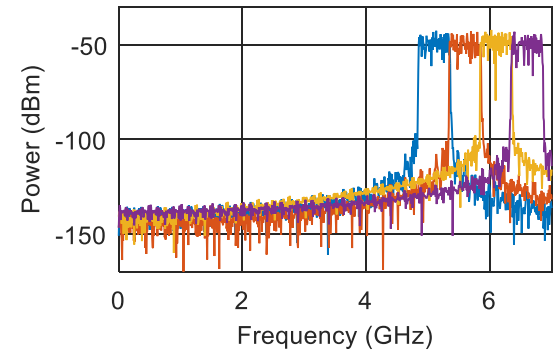

(a)

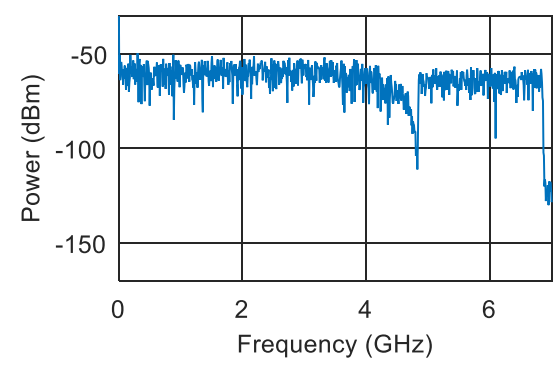

(b)

Fig. 2. Offline generated spectra of (a) 4 sub-bands FBMC (b) multiplexed 4-PAM and 4 sub-bands FBMC signal.

\section{Optical Transmission System}

The idea of the proposed method of wired-wireless convergence in PON system is given in Fig. 3. The setup consists of optical line terminal (OLT) and optical network unit (ONU) connected through an optical fiber. In the OLT, the 4-PAM and multi-sub-bands FBMC composite signal is amplified and fed to the intensity modulator (IM) and sent through an optical fiber. In the ONU, the received composite signal is detected by the photodetector and processed offline using DSP techniques for demodulation. The typical PON system includes power splitter in ONU and fiber between OLT and ONU. The power splitter cannot separate individual signals from converged signal carried by fiber. To separate the individual signals through converged signal after photodetector one need to employ analog electrical filter with center frequency as corresponding signal's frequency. In our case, we use the digital bandpass filter to separate the signals in ONU.

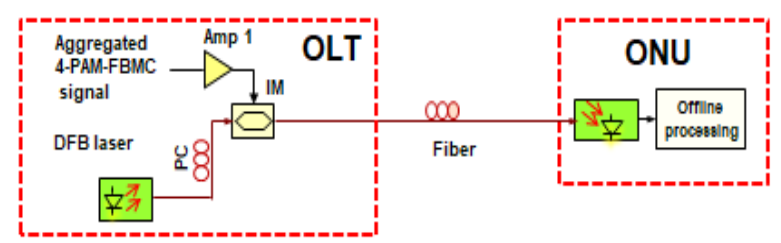

Fig. 3. Block diagram of PON setup with OLT and ONU.

VPItransmissionMaker simulator along with MATLAB cosimulation is used to implement and evaluate the performance with various design parameters. As shown in Fig. 4 (a), in the OLT setup, a continuous wave DFB laser is operated at $5 \mathrm{~mW}$ output power and wavelength of $1553.6 \mathrm{~nm}$. The linewidth of the laser is set to 10 MHz. The Mach Zehnder modulator (MZM) is a chirp less modulator. The half wave voltage of MZM is at $8 \mathrm{~V}$. The driving signal for modulator is a composite 4-PAM and multi-sub-bands
FBMC signal which is generated offline developing MATLAB routines as described in section II. The MZM is biased at the quadrature point. The modulated optical signal is then transmitted through the optical fiber. The used optical fiber is a standard single mode fiber (SMF). The fiber dispersion is $18 \mathrm{ps} /(\mathrm{nm} \mathrm{km})$.

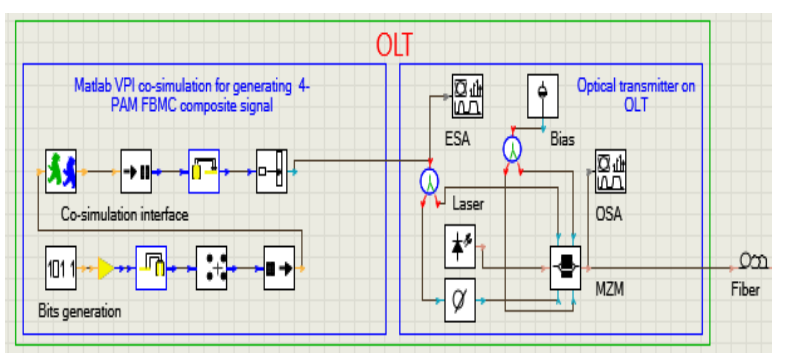

(a)

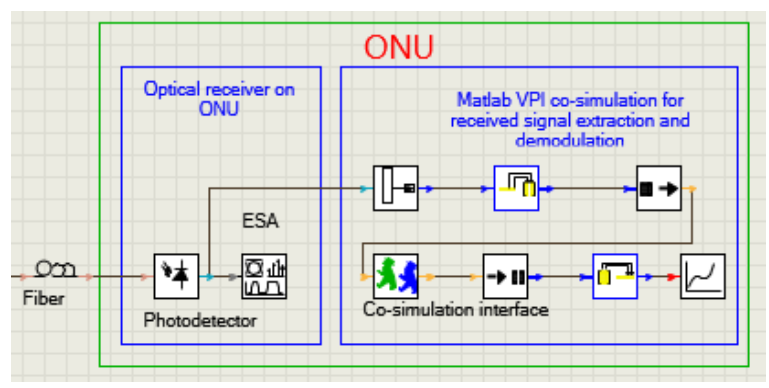

(b)

Fig. 4. Design of PON setup in VPItransmissionMaker simulator with MATLAB co-simulation. (a) OLT (b) ONU. ESA: electrical spectrum analyzer, OSA : optical spectrum analyzer, MZM: Mach Zehnder modulator

As shown in Fig. 4 (b), in the ONU, the signal is detected with positive intrinsic negative $(\mathrm{p}-\mathrm{i}-\mathrm{n})$ photodiode with a thermal noise parameter of $10^{-12} \mathrm{pA} / \mathrm{Hz}^{1 / 2}$. The signal is then processed offline using MATLAB routines. Fig. 5 shows the generated optical spectrum after MZM in OLT, which shows the aggregated baseband 4-PAM signal along with the multi-sub-bands FBMC signal in the optical double sidebands.

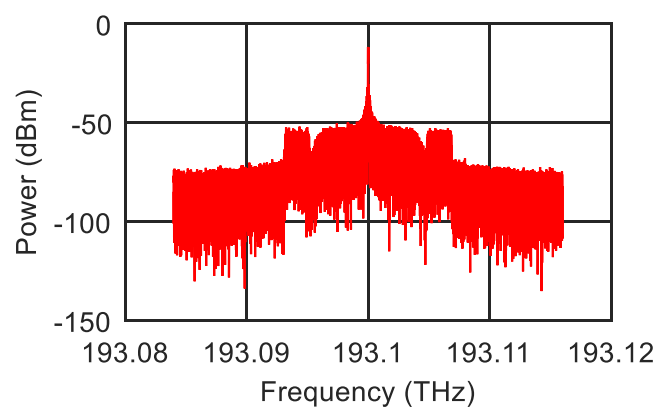

Fig. 5. Generated optical spectrum after MZM in OLT.

\section{DSP RECEIVER OfFLINE PROCESSING}

The received signal after photodetector in ONU is captured at 32 GS/s whose spectrum is shown in Fig. 6. The 4-PAM and multi-subbands FBMC signals are extracted and demodulated separately. For 
Wired-Wireless Converged Passive Optical Network

with 4-PAM and Multi-sub-bands FBMC

the multi-sub-bands FBMC, the signal at baseband is achieved after downconverting with the corresponding sub-bands intermediate frequency (IF) along with the RRC low pass filtering. After this, the resampling is applied to downsample the signal at 2 samples for each OQAM symbols. The FBMC decoding routines are applied as shown in Fig. 7, as explained in $[10,13]$. The decoding process involves $\mathrm{S} / \mathrm{P}$ conversion, analysis filter bank, FFT, OQAM-QAM conversion and $\mathrm{P} / \mathrm{S}$ conversion. The training length of first 16 QAM samples from each sub-bands are used to calculate the channel response which is used to equalize the signal. Furthermore, simple 1 tap recursive least square (RLS) equalizer with forgetting factor of 0.9 is used to optimize the equalization process. After the equalization, the performance of the signal is evaluated with BER calculation.

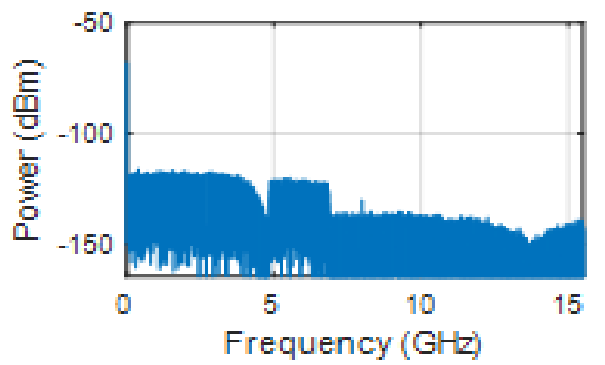

Fig. 6. Received photo-detected signal in ONU after $40 \mathrm{~km}$ fiber length.

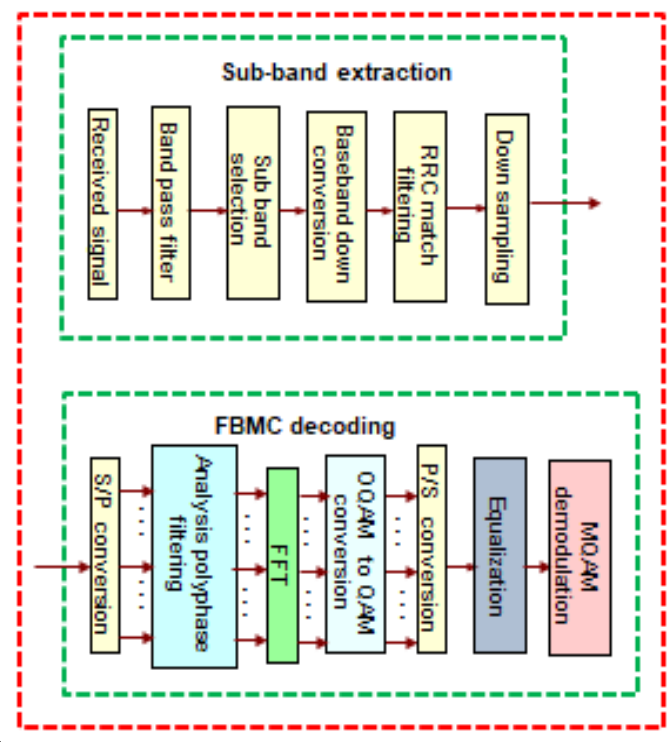

Fig. 7. FBMC sub-bands extraction and decoding.

Fig. 8 (a) describes the 4-PAM signal extraction and demodulation steps in ONU. Square bandpass filter with center frequency at $0 \mathrm{~Hz}$ and bandwidth of $9.6 \mathrm{GHz}$ is applied to the received signal which extracts the two sidebands of the received 4-PAM signal as shown in Fig. 8 (b). Thus extracted 4-PAM signal is equalized with the 9 taps adaptive feed forward finite impulse response (FIR) equalizer (FFE) with least mean square (LMS) adaptation. The first 64 samples of the 4-PAM signal are used as a training length for the adaptation of LMS algorithm. After equalization, the performance of the 4-PAM signal is evaluated with BER calculation.

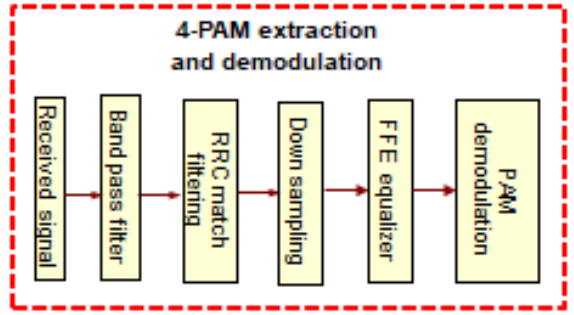

(a)

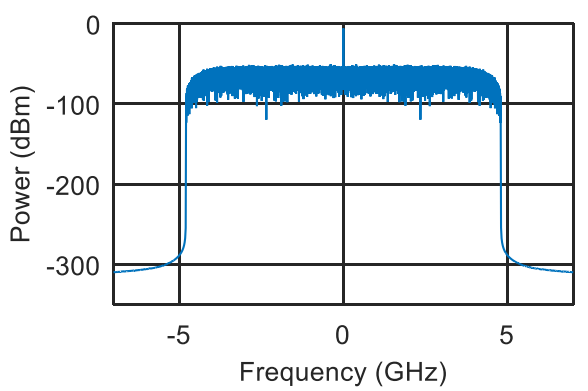

(b)

Fig. 8. (a) 4-PAM signal extraction and demodulation process. (b) Extracted 4-PAM signal.

\section{RESUlts AND Discussions}

Fig. 9 shows the BER versus received optical power (ROP) performance for separate transmission of 4-PAM and 4 sub-bands FBMC at the fiber length of $40 \mathrm{~km}$ and $10 \mathrm{MHz}$ laser linewidth.

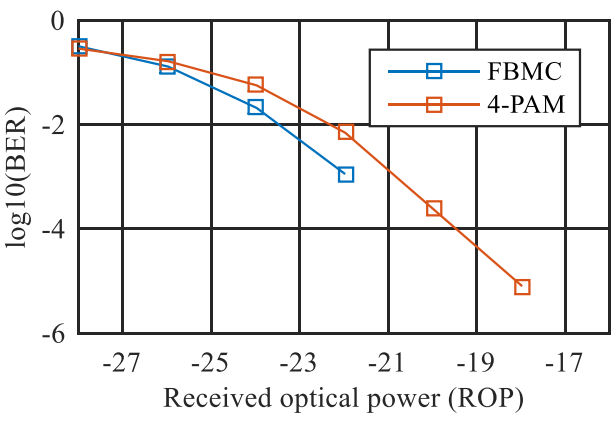

Fig. 9. BER versus ROP performance of separate 4-PAM and 4 sub-bands FBMC signal transmission.

If 4-PAM and FBMC signals are separately transmitted (without mixing), for the equivalent design parameters, FBMC shows better performance compared to the 4-PAM as shown in Fig. 9. The BER of $10^{-3}$ can be obtained at ROP of $-22 \mathrm{dBm}$ for the case of FBMC and $-21 \mathrm{dBm}$ for the case of 4-PAM. For the ROP values greater than $-22 \mathrm{dBm}$, the BER of FBMC becomes 0 . Similarly, for the ROP values greater than $-18 \mathrm{dBm}$, the BER of 4-PAM becomes 0 . From this simulation result, it can be concluded that the 4 subbands FBMC shows the better performance than 4-PAM for the equivalent design parameters.

The 4 sub-bands FBMC and 4-PAM signals are now multiplexed and the composite signal is transmitted. The 4-PAM and FBMC 
signals were extracted separately from the received composite signal using square bandpass filter. The BER with different received optical power (ROP) is evaluated as shown in Fig. 10 with $10 \mathrm{MHz}$ linewidth and $40 \mathrm{~km}$ fiber length for the extracted 4-PAM and 4 sub-bands FBMC signals. By varying ROP, for lower values of ROP, both modulation schemes show the similar performance. For the ROP of $-16 \mathrm{dBm}$, the BER of $10^{-3}$ can be obtained for both the modulation schemes. As the ROP increases the 4-PAM signal shows better performance than 4 sub-bands FBMC signal indicating the fact that FBMC is more affected by the signal mixing effect (interference) compared to 4-PAM in the composite signal case.

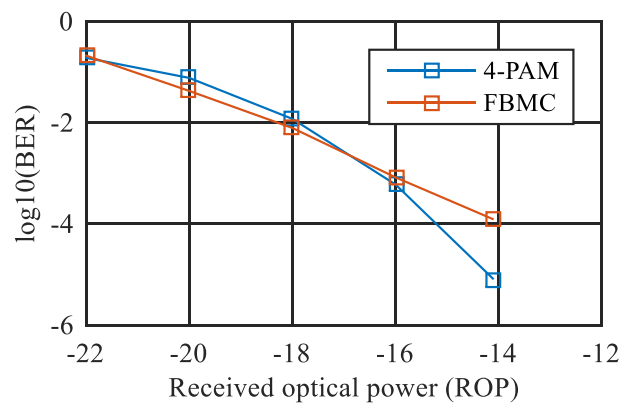

Fig. 10. BER versus ROP performances of 4-PAM and FBMC signals.

The BER versus fiber length performance is evaluated for the extracted 4-PAM and 4 sub-bands FBMC signal with the laser linewidth of $10 \mathrm{MHz}$ as shown in Fig. 11. The FBMC has degraded performance compared to 4-PAM. In this simulation also, in the case of the composite signal, the FBMC signal is more affected by the interference compared to the 4-PAM signal.

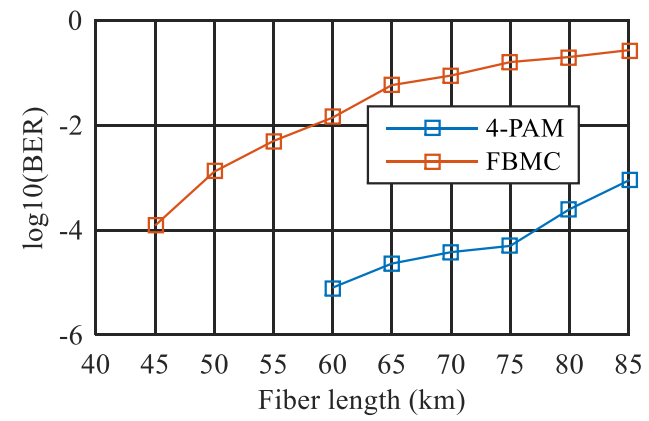

Fig. 11. BER versus fiber length performances of 4-PAM and 4 sub-bands FBMC signals.

Fig. 12 (a) and (b) show the constellation diagrams of the extracted 4 sub-bands FBMC and 4-PAM signals at $40 \mathrm{~km}$ and 60 $\mathrm{km}$ fiber lengths respectively. At $40 \mathrm{~km}$ fiber length, the constellations of the 4 sub-bands FBMC and 4-PAM signals are less distorted which corresponds to the BER of less than $10^{-6}$ for both the modulation schemes. As the fiber length increases the constellation of the FBMC is distorted more. For the $60 \mathrm{~km}$ fiber length, the 4-PAM signal BER is in the order of $10^{-5}$ as compared to $10^{-2}$ for the case of 4 sub-bands FBMC.
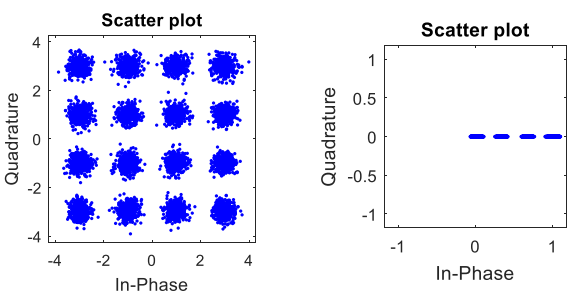

(a)
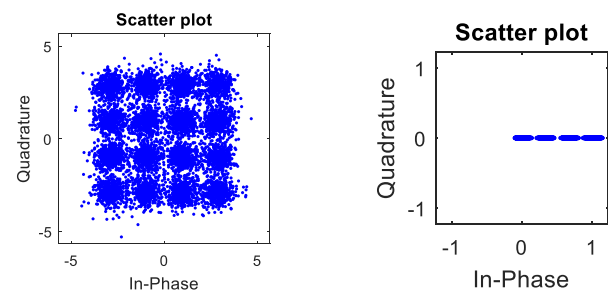

(b)

Fig. 12. Constellation diagrams of extracted 4 sub-bands FBMC and 4-PAM signals after (a) $40 \mathrm{~km}$ fiber length (b) $60 \mathrm{~km}$ fiber length.

\section{CONCLUSION}

In this paper, by using FBMC signal of 4 sub-bands of $500 \mathrm{MHz}$ each with the aggregated bandwidth of $2.0015 \mathrm{GHz}$ and 4-PAM of $4.8 \mathrm{GHz}$ we demonstrated the performance of converged wiredwireless signal transmission in the PON. The 4 sub-bands FBMC signal has the bit rate of $4 \mathrm{Gbps}$ and 4-PAM has the bit rate of 8 Gbps. The narrow-band gap of $488.28 \mathrm{kHz}$ is used to separate each sub-bands in 4 sub-bands FBMC. With very low-performance degradation $\left(\mathrm{BER}<10^{-6}\right.$ ) the converged signal can be transmitted for a distance of up to $40 \mathrm{~km}$ for the received optical power (ROP) of $4.1 \mathrm{dBm}$ with the laser linewidth of $10 \mathrm{MHz}$. The BER of $10^{-3}$ can be obtained at ROP of $-22 \mathrm{dBm}$ for the case of 4 sub-bands FBMC and $-21 \mathrm{dBm}$ for the case of 4-PAM at $40 \mathrm{~km}$ fiber length and laser linewidth of $10 \mathrm{MHz}$.

The FBMC shows better performance compared to 4-PAM for separate transmission. However, FBMC is more affected by the laser linewidth and the interference effect compared to 4-PAM in the converged signal transmission scenario. Also, due to the double bandwidth efficiency of the 4-PAM, it will be attractive candidate compared to conventional OOK in baseband signal transmission scenario. We believe that the convergence of multi-sub-bands FBMC and 4-PAM will make expected 5G high bandwidth multi-user, multibands wireless services feasible.

\section{REFERENCES}

[1] J. Y. Sung, C. W. Chow, C. H. Yeh, Y. Liu and G. K. Chang, "Costeffective mobile backhaul network using existing ODN of PONs for the $5 \mathrm{G}$ wireless systems," in IEEE Photonics Journal, vol. 7, no. 6, pp. 1-6, Dec. 2015. doi: 10.1109/JPHOT.2015.2497222.

[2] B. Skubic, M. Fiorani, S. Tombaz, A. Furuskär, J. Mårtensson and P. Monti, "Optical transport solutions for $5 \mathrm{G}$ fixed wireless access [Invited]," in IEEE/OSA Journal of Optical Communications and Networking, vol. 9, no. 9, pp. D10-D18, Sept.2017.doi:10.1364/JOCN.9.000D10 
[3] X. Liu and F. Effenberger, "Emerging optical access network technologies for 5G wireless [invited]," in IEEE/OSA Journal of Optical Communications and Networking, vol. 8, no. 12, pp. B70-B79,2016. doi:10.1364/JOCN.8.000B70.

[4] T. S. Rappaport et al., "Millimeter wave mobile communications for 5G cellular: It will work!," in IEEE Access, vol.1, pp.335-349, 2013. doi: 10.1109/ACCESS.2013.2260813

[5] A. Tzanakaki et al., "Wireless-optical network convergence: enabling the $5 \mathrm{G}$ architecture to support operational and end-user services," in IEEE Communications Magazine, vol. 55, no. 10, pp. 184-192, OCTOBER 2017. doi: 10.1109/MCOM.2017.1600643.

[6] B. Skubic, M. Fiorani, S. Tombaz, A. Furuskär, J. Mårtensson and P. Monti, "Optical transport solutions for $5 \mathrm{G}$ fixed wireless access [Invited]," in IEEE/OSA Journal of Optical Communications and Networking, vol. 9, no. 9, pp. D10-D18, Sept. 2017. doi: 10.1364/JOCN.9.000D10.

[7] E. P. Martin et al., "25-Gb/s OFDM 60-GHz radio over fiber system based on a gain switched laser," in Journal of Lightwave Technology, vol. 33 , no. 8, pp. 1635-1643, April15, , 2015. doi: 10.1109/JLT.2015.2391994.

[8] H. Shams and J. Zhao, "First investigation of fast OFDM radio over fibre system at $60 \mathrm{GHz}$ using direct laser modulation," 2013 Conference on Lasers \& Electro-Optics Europe \& International Quantum Electronics Conference CLEO EUROPE/IQEC, Munich, 2013, pp. 1-1.

[9] P. Banelli, S. Buzzi, G. Colavolpe, A. Modenini, F. Rusek and A. Ugolini, "Modulation formats and waveforms for $5 \mathrm{G}$ networks: who will be the heir of OFDM?: An overview of alternative modulation schemes for improved spectral efficiency," in IEEE Signal Processing Magazine, vol. 31, no. 6, pp. 80-93, Nov. 2014. doi: 10.1109/MSP.2014.2337391

[10] J. Zhang et al., "Full-duplex quasi-gapless carrier aggregation using FBMC in centralized radio-over-fiber heterogeneous networks," in Journal of Lightwave Technology, vol. 35, no. 4, pp. 989-996, Feb.15, 15 2017. doi: 10.1109/JLT.2016.2608138.

[11] T. T. Nguyen, S. T. Le, Q. He, L. V. Compernolle, M. Wuilpart and P. Mégret, "Multicarrier approaches for high-baudrate optical-fiber transmission systems with a single coherent receiver," in IEEE Photonics Journal, vol. 9, no. 2, pp. 1-10, 2017. doi: 10.1109/JPHOT.2017.2672041.

[12] M. Xu et al., "FBMC in next-generation mobile fronthaul networks with centralized pre-equalization," in IEEE Photonics Technology Letters, vol. 28, no. 18, pp. 1912-1915, Sept.15, 15 2016. doi: 10.1109/LPT.2016.2575060

[13] M. Bellanger, D. Le Ruyet, et al.,"FBMC physical layer: a primer," PHYDYAS, Project Document, Jan. (2010). [Online available: http://www.ict-phydyas.org/]
[14] I. Lazarou, S. Dris, P. Bakopoulos, B. Schrenk and H. Avramopoulos, "Full-duplex 4-PAM transmission for capacity upgrade in loop-back PONs," in IEEE Photonics Technology Letters, vol. 25, no. 12, pp. 1125-1128, June 15, 2013. doi: 10.1109/LPT.2013.2260533.

[15] H. K. Shim, H. Kim and Y. C. Chung, "20-Gb/s polar RZ 4PAM transmission over 20-km SSMF using RSOA and direct detection," in IEEE Photonics Technology Letters, vol. 27, no. 10, pp. 1116-1119, May 15, 2015. doi: 10.1109/LPT.2015.2408376.

[16] C. Stamatiadis, R. Matsumoto, Y. Yoshida, A. Agata, A. Maruta and K. I. Kitayama, "Full-duplex RSOA-based PONs using 4PAM with pre-equalization," in IEEE Photonics Technology Letters, vol. 27, no. 1, pp. 73-76, Jan.1,2015. doi: 10.1109/LPT.2014.2361922.

[17] A. Saljoghei, F. A. Gutiérrez, P. Perry, D. Venkitesh, R. D. Koipillai and L. P. Barry, "Experimental comparison of FBMC and OFDM for multiple access uplink PON," in Journal of Lightwave Technology, vol.

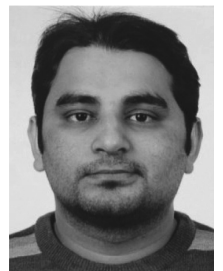

Hum Nath Parajuli is a Marie Curie early stage researcher in Budapest University of Technology and Economics, Budapest, Hungary. He received his B.Eng. degree from Pokhara University, Nepal in 2008 and joint M.Sc. degree from Osaka University, Japan and Scula Superiore Sant' Anna, Italy in 2012 His current research interests include design of high capacity optical wireless links, millimeter wave communication, digital signal processing for optical communication and design of optoelectronic systems.

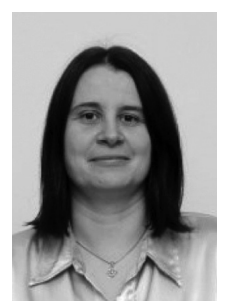

Eszter Udvary received Ph.D. degree in electrical engineering from Budapest University of Technology and Economics (BME), Budapest, Hungary, in 2009. She is currently an Associate Professor at BME, Department of Broadband Info-communications and Electromagnetic Theory, where she leads the Optical and Microwave Telecommunication Lab. Dr. Udvary's research interests are in the broad areas of optical communications, include optical and microwave communication systems, Radio over applications of special electro-optical devices. 